\title{
Left localizable rings and their characterizations
}

\author{
V. V. Bavula
}

\begin{abstract}
A new class of rings, the class of left localizable rings, is introduced. A ring $R$ is left localizable if each nonzero element of $R$ is invertible in some left localization $S^{-1} R$ of the ring $R$. Explicit criteria are given for a ring to be a left localizable ring provided the ring has only finitely many maximal left denominator sets (eg, this is the case if a ring has a left Artinian left quotient ring). It is proved that a ring with finitely many maximal left denominator sets is a left localizable ring iff its left quotient ring is a direct product of finitely many division rings. A characterization is given of the class of rings that are finite direct product of left localization maximal rings.

Key Words: a left localizable ring, a left localization maximal ring, the largest left quotient ring of a ring, the largest regular left Ore set of a ring, the classical left quotient ring of a ring, denominator set.

Mathematics subject classification 2000: 16U20, 16P50, $16 S 85$.
\end{abstract}

\section{Contents}

1. Introduction.

2. Direct products of left localization maximal rings and their characterization.

3. Left localizable rings and their characterization.

4. The core of a left Ore set.

\section{Introduction}

Throughout, module means a left module. In this paper the following notation will remained fixed.

\section{Notation:}

- $R$ is a ring with 1 and $\mathcal{C}_{R}$ is the set of (left and right) regular elements of the ring $R$ (i.e. $\mathcal{C}_{R}$ is the set of non-zero-divisors of $R$ );

- $Q_{l, c l}(R):=\mathcal{C}_{R}^{-1} R$ is the classical left quotient ring of $R$ (if it exists);

- $\operatorname{Ore}_{l}(R):=\{S \mid S$ is a left Ore set in $R\}$;

- $\operatorname{Den}_{l}(R):=\{S \mid S$ is a left denominator set in $R\}$;

- $\operatorname{Loc}_{l}(R):=\left\{\left[S^{-1} R\right] \mid S \in \operatorname{Den}_{l}(R)\right\}$ where $\left[S^{-1} R\right]$ is an $R$-isomorphism class of the ring $S^{-1} R$ (a ring isomorphism $\sigma: S^{-1} R \rightarrow S^{-1} R$ is called an $R$-isomorphism if $\sigma\left(\frac{r}{1}\right)=\frac{r}{1}$ for all elements $r \in R$ );

- $\mathfrak{l}_{R}:=1 . \operatorname{lrad}(R)$ is the left localization radical of the $\operatorname{ring} R$;

- $\operatorname{Ass}_{l}(R):=\left\{\operatorname{ass}(S) \mid S \in \operatorname{Den}_{l}(R)\right\}$ where $\operatorname{ass}(S):=\{r \in R \mid s r=0$ for some $s=s(r) \in S\}$;

- $\operatorname{Den}_{l}(R, \mathfrak{a}):=\left\{S \in \operatorname{Den}_{l}(R) \mid \operatorname{ass}(S)=\mathfrak{a}\right\}$ where $\mathfrak{a} \in \operatorname{Ass}_{l}(R)$;

- $\operatorname{Den}_{l}(R, 0)$ is the set of regular left denominator sets $S$ in $R\left(S \subseteq \mathcal{C}_{R}\right)$; 
- $S_{\mathfrak{a}}=S_{\mathfrak{a}}(R)=S_{l, \mathfrak{a}}(R)$ is the largest element of the poset $\left(\operatorname{Den}_{l}(R, \mathfrak{a}), \subseteq\right)$ and $Q_{\mathfrak{a}}(R):=$ $Q_{l, \mathfrak{a}}(R):=S_{\mathfrak{a}}^{-1} R$ is the largest left quotient ring associated to $\mathfrak{a}, S_{\mathfrak{a}}$ exists (Theorem 2.1, [2]);

- In particular, $S_{0}=S_{0}(R)=S_{l, 0}(R)$ is the largest element of the poset $\left(\operatorname{Den}_{l}(R, 0), \subseteq\right)$ and $Q_{l}(R):=S_{0}^{-1} R$ is the largest left quotient ring of $R$;

- $\operatorname{Loc}_{l}(R, \mathfrak{a}):=\left\{\left[S^{-1} R\right] \mid S \in \operatorname{Den}_{l}(R, \mathfrak{a})\right\}$.

Left localizable rings and their characterization. The aim of the paper is to introduce a new class of rings, the class of left localizable rings, and to give several characterizations of them (Theorem 3.5, Theorem 3.8 and Theorem 3.9) in the case when they admit only finitely many maximal left denominator sets. Notice that each ring with left Artinian, left quotient ring has only finitely many maximal left denominator sets, 4. A ring $R$ is called a left localizable ring if for each nonzero element $r \in R$ there exists a left denominator set $S=S(r)$ such that $r \in S$ (equivalently, if for each nonzero element $r \in R$ there exists a left denominator set $S^{\prime}=S^{\prime}(r)$ such that $\frac{r}{1}$ is a unit in $S^{\prime-1} R$ ).

- (Theorem 3.5) Let $R$ be a ring with $\max \cdot \operatorname{Den}_{l}(R)=\left\{S_{1}, \ldots, S_{n}\right\}$. Let $\mathfrak{a}_{i}:=\operatorname{ass}\left(S_{i}\right)$; $\sigma_{i}: R \rightarrow R_{i}:=S_{i}^{-1} R, r \mapsto \frac{r}{1}=r_{i}$; and $\sigma:=\prod_{i=1}^{n} \sigma_{i}: R \rightarrow \prod_{i=1}^{n} R_{i}, r \mapsto\left(r_{1}, \ldots, r_{n}\right)$. The following statements are equivalent.

1. The ring $R$ is a left localizable ring.

2. $\mathfrak{l}_{R}=0$ and the rings $R_{1}, \ldots, R_{n}$ are division rings.

3. The homomorphism $\sigma$ is an injection and the rings $R_{1}, \ldots, R_{n}$ are division rings.

- (Theorem (3.9) Let $R$ be a ring. The following statements are equivalent.

1. The ring $R$ is a left localizable ring with $n:=\left|\max \cdot \operatorname{Den}_{l}(R)\right|<\infty$.

2. $Q_{l, c l}(R)=R_{1} \times \cdots \times R_{n}$ where $R_{i}$ are division rings.

3. The ring $R$ is a semiprime left Goldie ring with $\operatorname{udim}(R)=|\operatorname{Min}(R)|=n$ where $\operatorname{Min}(R)$ is the set of minimal prime ideals of the ring $R$.

4. $Q_{l}(R)=R_{1} \times \cdots \times R_{n}$ where $R_{i}$ are division rings.

Direct product of left localization maximal rings and their characterization. A ring $R$ is called a left localization maximal ring if every left denominator set of $R$ consists of units, i.e. one cannot invert anything new 'on the left' in $R, 3$. Theorem 2.11 is a characterization of rings that are isomorphic to finite direct product of left localization maximal rings.

- (Theorem 2.11) $A$ ring $R$ is a direct product of $n$ left localization maximal rings iff the following conditions hold:

1. $\max \cdot \operatorname{Den}_{l}(R)=\left\{S_{1}, \ldots, S_{n}\right\}$.

2. $\mathfrak{l}_{R}=0$.

3. $\operatorname{ass}\left(S_{i}\right)+\operatorname{ass}\left(S_{j}\right)=R$ for all $i \neq j$.

4. The factor rings $R / \operatorname{ass}\left(S_{i}\right), i=1, \ldots, n$, are left localization maximal rings.

The core of a left Ore set. In Section 4 , properties of the core of a left Ore set are studied. Let $R$ be a ring and $S$ be its left Ore set. The subset of $S$,

$$
S_{c}:=\{s \in S \mid \operatorname{ker}(s \cdot)=\operatorname{ass}(S)\}
$$

is called the core of the left Ore set $S$ where $s \cdot R \rightarrow R, r \mapsto s r$, [3].

- (Theorem 4.2) Suppose that $S \in \operatorname{Den}_{l}(R, \mathfrak{a})$ and $S_{c} \neq \emptyset$. Then 
1. $S_{c} \in \operatorname{Den}_{l}(R, \mathfrak{a})$.

2. The map $\theta: S_{c}^{-1} R \rightarrow S^{-1} R, s^{-1} r \mapsto s^{-1} r$, is a ring isomorphism. So, $S_{c}^{-1} R \simeq S^{-1} R$.

The next theorem gives an explicit description of the cores of maximal left denominator sets of a left localizable ring provided $\left|\max \operatorname{Den}_{l}(R)\right|<\infty$.

- (Theorem 4.5) Let $R$ be a left localizable ring such that $\max \cdot \operatorname{Den}_{l}(R)=\left\{S_{1}, \ldots, S_{n}\right\}$.

1. If $n=1$ then $S_{1, c}=S_{1}=R \backslash\{0\}$.

2. If $n \geq 2$ then $S_{i, c}=S_{i} \cap \bigcap_{j \neq i} \mathfrak{a}_{i}$ where $\mathfrak{a}_{j}=\operatorname{ass}\left(S_{j}\right)$.

\section{Direct products of left localization maximal rings and their characterization}

The aim of this section is to give a criterion of when a ring is isomorphic to a finite direct product of left localization maximal rings (Theorem 2.11). At the beginning of the section necessary results are given that are used in the proofs of the paper.

In [2, we introduce the following new concepts and to prove their existence for an arbitrary ring: the largest left quotient ring of a ring, the largest regular left Ore set of a ring, the maximal left quotient rings of a ring, the largest (two-sided) quotient ring of a ring, the maximal (two-sided) quotient rings of a ring, a (left) localization maximal ring. Let us give more details.

The largest regular left Ore set and the largest left quotient ring of a ring. Let $R$ be a ring. A multiplicatively closed subset $S$ of $R$ or a multiplicative subset of $R$ (i.e. a multiplicative sub-semigroup of $(R, \cdot)$ such that $1 \in S$ and $0 \notin S)$ is said to be a left Ore set if it satisfies the left Ore condition: for each $r \in R$ and $s \in S$,

$$
\operatorname{Sr} R s \neq \emptyset .
$$

Let $\operatorname{Ore}_{l}(R)$ be the set of all left Ore sets of $R$. For $S \in \operatorname{Ore}_{l}(R), \operatorname{ass}(S):=\{r \in R \mid s r=$ 0 for some $s \in S\}$ is an ideal of the ring $R$.

A left Ore set $S$ is called a left denominator set of the $\operatorname{ring} R$ if $r s=0$ for some elements $r \in R$ and $s \in S$ implies $t r=0$ for some element $t \in S$, i.e. $r \in \operatorname{ass}(S)$. Let $\operatorname{Den}_{l}(R)$ be the set of all left denominator sets of $R$. For $S \in \operatorname{Den}_{l}(R)$, let $S^{-1} R=\left\{s^{-1} r \mid s \in S, r \in R\right\}$ be the left localization of the ring $R$ at $S$ (the left quotient ring of $R$ at $S$ ).

In general, the set $\mathcal{C}$ of regular elements of a ring $R$ is neither left nor right Ore set of the ring $R$ and as a result neither left nor right classical quotient ring $\left(Q_{l, c l}(R):=\mathcal{C}^{-1} R\right.$ and $Q_{r, c l}(R):=$ $R C^{-1}$ ) exists. Remarkably, there exists the largest regular left Ore set $S_{0}=S_{l, 0}=S_{l, 0}(R)$. This means that the set $S_{l, 0}(R)$ is an Ore set of the ring $R$ that consists of regular elements (i.e., $\left.S_{l, 0}(R) \subseteq \mathcal{C}\right)$ and contains all the left Ore sets in $R$ that consist of regular elements. Also, there exists the largest regular (left and right) Ore set $S_{l, r, 0}(R)$ of the ring $R$. In general, all the sets $\mathcal{C}$, $S_{l, 0}(R), S_{r, 0}(R)$ and $S_{l, r, 0}(R)$ are distinct, for example, when $R=\mathbb{I}_{1}:=K\left\langle x, \frac{d}{d x}, \int\right\rangle$ is the ring of polynomial integro-differential operators over a field $K$ of characteristic zero, [1].

Definition, [1], 2]. The ring

$$
Q_{l}(R):=S_{l, 0}(R)^{-1} R
$$

(respectively, $Q_{r}(R):=R S_{r, 0}(R)^{-1}$ and $Q(R):=S_{l, r, 0}(R)^{-1} R \simeq R S_{l, r, 0}(R)^{-1}$ ) is called the largest left (respectively, right and two-sided) quotient ring of the ring $R$.

In general, the rings $Q_{l}(R), Q_{r}(R)$ and $Q(R)$ are not isomorphic, for example, when $R=\mathbb{I}_{1}$, [1. The next theorem gives various properties of the ring $Q_{l}(R)$. In particular, it describes its group of units.

Theorem 2.1 [2] 
1. $S_{0}\left(Q_{l}(R)\right)=Q_{l}(R)^{*}$ and $S_{0}\left(Q_{l}(R)\right) \cap R=S_{0}(R)$.

2. $Q_{l}(R)^{*}=\left\langle S_{0}(R), S_{0}(R)^{-1}\right\rangle$, i.e. the group of units of the ring $Q_{l}(R)$ is generated by the sets $S_{0}(R)$ and $S_{0}(R)^{-1}:=\left\{s^{-1} \mid s \in S_{0}(R)\right\}$.

3. $Q_{l}(R)^{*}=\left\{s^{-1} t \mid s, t \in S_{0}(R)\right\}$.

4. $Q_{l}\left(Q_{l}(R)\right)=Q_{l}(R)$.

The set $\left(\operatorname{Den}_{l}(R), \subseteq\right)$ is a poset (partially ordered set). In [2], it is proved that the set max.Den ${ }_{l}(R)$ of its maximal elements is a non-empty set.

The maximal denominator sets and the maximal left localizations of a ring.

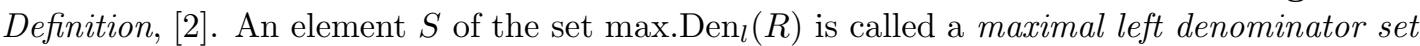
of the ring $R$ and the ring $S^{-1} R$ is called a maximal left quotient ring of the ring $R$ or a maximal left localization ring of the ring $R$. The intersection

$$
\mathfrak{l}_{R}:=1 \cdot \operatorname{lrad}(R):=\bigcap_{S \in \max _{\operatorname{man}}(R)} \operatorname{ass}(S)
$$

is called the left localization radical of the ring $R,[2$.

For a ring $R$, there is the canonical exact sequence

$$
0 \rightarrow \mathfrak{l}_{R} \rightarrow R \stackrel{\sigma}{\rightarrow} \prod_{S \in \max \operatorname{Den}_{l}(R)} S^{-1} R, \quad \sigma:=\prod_{S \in \max ^{-\operatorname{Den}_{l}(R)}} \sigma_{S},
$$

where $\sigma_{S}: R \rightarrow S^{-1} R, r \mapsto \frac{r}{1}$.

The maximal elements of $\operatorname{Ass}_{l}(R)$. Let max. $\operatorname{Ass}_{l}(R)$ be the set of maximal elements of the poset $\left(\operatorname{Ass}_{l}(R), \subseteq\right)$ and

$$
\operatorname{ass.max.} \operatorname{Den}_{l}(R):=\left\{\operatorname{ass}(S) \mid S \in \max \operatorname{Den}_{l}(R)\right\} .
$$

These two sets are equal (Proposition 2.4), a proof is based on Lemma 2.2, For a non-empty subset $X$ of $R$, let $\operatorname{rass}(X):=\{r \in R \mid r x=0$ for some $x \in X\}$.

Lemma 2.2 [2] Let $S \in \operatorname{Den}_{l}(R, \mathfrak{a})$ and $T \in \operatorname{Den}_{l}(R, \mathfrak{b})$ be such that $\mathfrak{a} \subseteq \mathfrak{b}$. Let $S T$ be the multiplicative semigroup generated by $S$ and $T$ in $(R, \cdot)$. Then

1. $\operatorname{r} \cdot \operatorname{ass}(S T) \subseteq \mathfrak{b}$.

2. $S T \in \operatorname{Den}_{l}(R, \mathfrak{c})$ and $\mathfrak{b} \subseteq \mathfrak{c}$.

Corollary 2.3 Let $R$ be a ring, $S \in \max ^{-D}(R)$ and $T \in \operatorname{Den}_{l}(R)$. Then $T \subseteq S$ iff $\operatorname{ass}(T) \subseteq$ $\operatorname{ass}(S)$.

Proof. $(\Rightarrow)$ If $T \subseteq S$ then $\operatorname{ass}(T) \subseteq \operatorname{ass}(S)$.

$(\Leftarrow)$ If $\operatorname{ass}(T) \subseteq \operatorname{ass}(S)$ then, by Lemma 2.2, $S T \in \operatorname{Den}_{l}(R)$ and $S \subseteq S T$, hence $S=S T$, by the maximality of $S$. Then $T \subseteq S$.

Proposition 2.4 [2] $\max \cdot \operatorname{Ass}_{l}(R)=\operatorname{ass} \cdot \max \cdot \operatorname{Den}_{l}(R) \neq \emptyset$. In particular, the ideals of this set are incomparable (i.e. neither $\mathfrak{a} \nsubseteq \mathfrak{b}$ nor $\mathfrak{a} \nsupseteq \mathfrak{b}$ ).

The localization maximal rings. The set $\left(\operatorname{Loc}_{l}(R), \rightarrow\right)$ is a partially ordered set (poset) where $A \rightarrow B$ if there is an $R$-homomorphism $A \rightarrow B$. There is no oriented loops in the poset $\operatorname{Loc}_{l}(R)$ apart from the $R$-isomorphism $A \rightarrow A$.

Let $\max \cdot \operatorname{Loc}_{l}(R)$ be the set of maximal elements of the poset $\left(\operatorname{Loc}_{l}(R), \rightarrow\right)$. Then (see [2]),

$$
\max \cdot \operatorname{Loc}_{l}(R)=\left\{S^{-1} R \mid S \in \max \cdot \operatorname{Den}_{l}(R)\right\}=\left\{Q_{l}(R / \mathfrak{a}) \mid \mathfrak{a} \in \operatorname{ass} \cdot \max \cdot \operatorname{Den}_{l}(R)\right\} .
$$


Definition, 22. A ring $A$ is called a left localization maximal ring if $A=Q_{l}(A)$ and $\operatorname{Ass}_{l}(A)=$ $\{0\}$. A ring $A$ is called a right localization maximal ring if $A=Q_{r}(A)$ and $\operatorname{Ass}_{r}(A)=\{0\}$. A ring $A$ which is a left and right localization maximal ring is called a (left and right) localization maximal ring (i.e. $Q_{l}(A)=A=Q_{r}(A)$ and $\operatorname{Ass}_{l}(A)=\operatorname{Ass}_{r}(A)=\{0\}$ ).

The next theorem is a criterion of when a left quotient ring of a ring is a maximal left quotient ring of the ring.

Theorem 2.5 [2] Let a ring $A$ be a left localization of a ring $R$, i.e. $A \in \operatorname{Loc}_{l}(R, \mathfrak{a})$ for some $\mathfrak{a} \in \operatorname{Ass}_{l}(R)$. Then $A \in \max _{L^{\prime}} \operatorname{Loc}_{l}(R)$ iff $Q_{l}(A)=A$ and $\operatorname{Ass}_{l}(A)=\{0\}$, i.e. A is a left localization maximal ring (clearly, $\mathfrak{a} \in$ ass.max.Den ${ }_{l}(R)$ ).

Theorem 2.5 shows that the left localization maximal rings are precisely the localizations of all the rings at their maximal left denominators sets.

Example. Let $A$ be a simple ring. Then $Q_{l}(A)$ is a left localization maximal ring and $Q_{r}(A)$ is a right localization maximal ring (by Theorem 2.1.(4) and Theorem 2.5).

Example. A division ring is a (left and right) localization maximal ring. More generally, a simple Artinian ring (i.e. the matrix algebra over a division ring) is a (left and right) localization maximal ring.

\section{Left (non-)localizable elements of a ring.}

Lemma 2.6 [4] Let $S \in \operatorname{Den}_{l}(R, \mathfrak{a})$ (respectively, $\left.S \in \operatorname{Den}(R, \mathfrak{a})\right), \sigma: R \rightarrow S^{-1} R, r \mapsto \frac{r}{1}$, and $G:=\left(S^{-1} R\right)^{*}$ be the group of units of the ring $S^{-1} R$. Then $S^{\prime}:=\sigma^{-1}(G) \in \operatorname{Den}_{l}(R, \mathfrak{a})$ (respectively, $S^{\prime}:=\sigma^{-1}(G) \in \operatorname{Den}(R, \mathfrak{a})$ ).

Definition, 3]. An element $r$ of a ring $R$ is called a left localizable element if there exists a left denominator set $S$ of $R$ such that $r \in S$ (and so the element $\frac{r}{1} \neq 0$ is invertible in the ring $S^{-1} R$ ), equivalently, if there exists a left denominator set $T$ of $R$ such that the element $\frac{r}{1}$ is invertible in the ring $T^{-1} R$ (Lemma 2.6). The set of left localizable elements is denoted $\mathcal{L}_{l}(R)$.

Clearly,

$$
\mathcal{L}_{l}(R)=\bigcup_{S \in \max _{\text {Den }}(R)} S
$$

Similarly, a right localizable element is defined and let $\mathcal{L}_{r}(R)$ be the set of right localizable elements of the ring $R$. The elements of the set of left and right localizable elements,

$$
\mathcal{L}_{l, r}(R)=\mathcal{L}_{l}(R) \cap \mathcal{L}_{r}(R),
$$

are called left and right localizable elements. An element $r \in R$ is called a localizable element if there exists a (left and right) denominator set $S \in \operatorname{Den}(R)$ such that $r \in S$, equivalently, if there exists a (left and right) denominator set $T \in \operatorname{Den}(R)$ such that the element $\frac{r}{1}$ is invertible in the ring $T^{-1} R$ (Lemma 2.6). The set of all localizable elements of the $\operatorname{ring} R$ is denoted by $\mathcal{L}(R)$. Clearly,

$$
\mathcal{L}(R) \subseteq \mathcal{L}_{l, r}(R)
$$

The sets

$$
\mathcal{N} \mathcal{L}_{l}(R):=R \backslash \mathcal{L}_{l}(R), \quad \mathcal{N} \mathcal{L}_{r}(R):=R \backslash \mathcal{L}_{r}(R), \quad \mathcal{N} \mathcal{L}_{l, r}(R):=R \backslash \mathcal{L}_{l, r}(R), \quad \mathcal{N} \mathcal{L}(R):=R \backslash \mathcal{L}(R),
$$

are called the sets of left non-localizable; of right non-localizable; of left and right non-localizable; of non-localizable elements, respectively. The elements of these sets are called correspondingly (eg, an element $r \in \mathcal{N} \mathcal{L}_{l}(R)$ is called a left non-localizable element).

Lemma 2.7 $\quad$ 1. $\mathfrak{l}_{R} \cap \mathcal{L}_{l}(R)=\emptyset$. 


\section{2. $\mathfrak{l}_{R} \subseteq \mathcal{N} \mathcal{L}_{l}(R)$.}

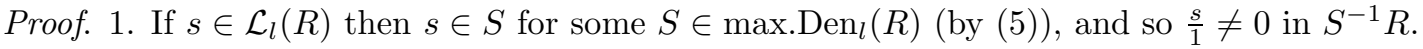
Therefore, $s \notin \mathfrak{l}_{R}$.

2. Statement 2 follows from statement 1 as $\mathcal{N} \mathcal{L}_{l}(R)=R \backslash \mathcal{L}_{l}(R)$.

Definition, [3. For an arbitrary ring $R$, the intersection

$$
\mathcal{C}_{l}(R):=\bigcap_{S \in \max ^{-\operatorname{Den}_{l}(R)}} S
$$

is called the set of completely left localizable elements of $R$ and an element of the set $\mathcal{C}_{l}(R)$ is called a completely left localizable element.

\section{The maximal left quotient rings of a finite direct product of rings.}

Theorem 2.8 [3] Let $R=\prod_{i=1}^{n} R_{i}$ be a direct product of rings $R_{i}$. Then for each $i=1, \ldots, n$, the map

$$
\max \cdot \operatorname{Den}_{l}\left(R_{i}\right) \rightarrow \max \cdot \operatorname{Den}_{l}(R), \quad S_{i} \mapsto R_{1} \times \cdots \times S_{i} \times \cdots \times R_{n},
$$

is an injection. Moreover, $\max _{\operatorname{Den}}(R)=\coprod_{i=1}^{n} \max _{l} \operatorname{Den}_{l}\left(R_{i}\right)$ in the sense of (6) , i.e.

$$
\max _{\operatorname{Den}}(R)=\left\{S_{i} \mid S_{i} \in \max _{l} \operatorname{Den}_{l}\left(R_{i}\right), i=1, \ldots, n\right\},
$$

$S_{i}^{-1} R \simeq S_{i}^{-1} R_{i}, \operatorname{ass}_{R}\left(S_{i}\right)=R_{1} \times \cdots \times \operatorname{ass}_{R_{i}}\left(S_{i}\right) \times \cdots \times R_{n}$. The core of the left denominator set $S_{i}$ in $R$ coincides with the core $S_{i, c}$ of the left denominator set $S_{i}$ in $R_{i}$, i.e.

$$
\left(R_{1} \times \cdots \times S_{i} \times \cdots \times R_{n}\right)_{c}=0 \times \cdots \times S_{i, c} \times \cdots \times 0 .
$$

Finite direct products of localization maximal rings. The next theorem describes properties of finite direct products of left localization maximal rings. It is used in the proof of Theorem 2.11 which is an explicit characterization of all such rings.

Theorem 2.9 Let $R_{1}, \ldots, R_{n}$ be left localization maximal rings and $R:=R_{1} \times \cdots \times R_{n}$. Then

1. $\max \cdot \operatorname{Den}_{l}(R)=\left\{S_{i} \mid i=1, \ldots, n\right\}$ and $S_{i}:=R_{1} \times \cdots \times R_{i}^{*} \times \cdots \times R_{n}$ where $R_{i}^{*}$ is a the group of units of the ring $R_{i}$.

2. $\operatorname{ass}\left(S_{i}\right)=R_{1} \times \cdots \times 0 \times \cdots \times R_{n}, i=1, \ldots, n$ where 0 is on $i^{\prime}$ th place; $\operatorname{ass}\left(S_{i}\right)+\operatorname{ass}\left(S_{j}\right)=R$ for all $i \neq j$.

3. $S_{i}^{-1} R \simeq R_{i} \simeq R / \operatorname{ass}\left(S_{i}\right), i=1, \ldots, n$. The map $\sigma_{i}: R \rightarrow S_{i}^{-1} R, r \mapsto \frac{r}{1}$, is equal to the map $R \rightarrow R / \operatorname{ass}\left(S_{i}\right), r \mapsto r+\operatorname{ass}\left(S_{i}\right)$.

4. $\mathfrak{l}_{R}=0$.

5. The natural ring homomorphism

$$
\sigma:=\prod_{I=1}^{n} \sigma_{i}: R \rightarrow \prod_{I=1}^{n} S_{i}^{-1} R, \quad r \mapsto\left(\frac{r}{1}, \ldots, \frac{r}{1}\right)=\left(r_{1}, \ldots, r_{n}\right),
$$

is an isomorphism.

6. $\mathcal{C}_{l}(R)=\bigcap_{i=1}^{n} S_{i}=R^{*}$ where $R^{*}=R_{1}^{*} \times \cdots \times R_{n}^{*}$ is the group of units of the ring $R$.

7. The set $\mathcal{L}_{l}(R)=\bigcup_{i=1}^{n} S_{i}$ of left localizable elements of the ring $R$ contains precisely the elements $\left(r_{1}, \ldots, r_{n}\right) \in R$ such that $r_{i} \in R_{i}^{*}$ for some $i$.

8. The set $\mathcal{N} \mathcal{L}_{l}(R)$ of left non-localizable elements of the ring $R$ is equal to $\prod_{i=1}^{n} R_{i} \backslash R_{i}^{*}$, the direct product of the sets of left non-localizable elements $\mathcal{N} \mathcal{L}_{l}\left(R_{i}\right)=R_{i} \backslash R_{i}^{*}$ of the rings $R_{i}$. 
Proof. 1. Statement 1 is a particular case of Theorem 2.8 .

$2-5$. For $i=1, \ldots, n$,

$$
S_{i} \in \max _{\operatorname{Den}}(R), \quad \operatorname{ass}\left(S_{i}\right)=R_{1} \times \cdots \times 0 \times \cdots \times R_{n}, \quad S_{i}^{-1} R \simeq R_{i} \simeq R / \operatorname{ass}\left(S_{i}\right) .
$$

and the localization map $\sigma_{i}$ is equal to the map $R \rightarrow R / \operatorname{ass}\left(S_{i}\right), r \mapsto r+\operatorname{ass}\left(S_{i}\right)$. Clearly, $\operatorname{ass}\left(S_{i}\right)+$ $\operatorname{ass}\left(S_{j}\right)=R$ for all $i \neq j$ and $\mathfrak{l}_{R} \subseteq \cap_{i=1}^{n}$ ass $\left(S_{i}\right)=0$, and so $\mathfrak{l}_{R}=0$. The ring homomorphism $\sigma$ is an isomorphism. So, statements $2-5$ have been proved.

6. Statement 6 follows from statement 1 .

7. Trivial.

8. Statement 8 follows from statement 1 .

Corollary 2.10 Let $R=\prod_{i=1}^{n} R_{i}$ be a direct product of left localization maximal rings $R_{i}$. Then every nonzero element of the ring $R$ is left localizable iff the ring $R_{1}, \ldots, R_{n}$ are division rings.

Proof. Every nonzero element of the ring $R$ is left localizable iff $\{0\}=\mathcal{N} \mathcal{L}_{l}(R)=\prod_{i=1}^{n} R_{i} \backslash R_{i}^{*}$ (Theorem 2.9. (8)) iff $R_{i} \backslash R_{i}^{*}=\{0\}$ for all $i$ iff the ring $R_{1}, \ldots, R_{n}$ are division rings.

Corollary 2.10 is a particular case of a criterion (Theorem 3.5) for a ring with finitely many maximal left denominator sets to be a left localizable ring.

Characterization of finite products of left localization maximal rings. The next theorem is a criterion for a ring to be isomorphic to a finite direct product of left localization maximal rings.

Theorem 2.11 $A$ ring $R$ is a direct product of $n$ left localization maximal rings iff the following conditions hold:

1. $\max \cdot \operatorname{Den}_{l}(R)=\left\{S_{1}, \ldots, S_{n}\right\}$.

2. $\mathfrak{l}_{R}=0$.

3. $\operatorname{ass}\left(S_{i}\right)+\operatorname{ass}\left(S_{j}\right)=R$ for all $i \neq j$.

4. The factor rings $R / \operatorname{ass}\left(S_{i}\right), i=1, \ldots, n$, are left localization maximal rings.

In this case,

(a) for each $i=1, \ldots, n$, the ring $R_{i}:=S_{i}^{-1} R$ is a left localization maximal ring such that the homomorphism $\sigma_{i}: R \rightarrow R_{i}, r \mapsto \frac{r}{1}$, is an epimorphism with $\operatorname{ker}\left(\sigma_{i}\right)=\operatorname{ass}\left(S_{i}\right)$, and so $R_{i} \simeq R / \operatorname{ass}\left(S_{i}\right)$.

(b) The map $\sigma:=\prod_{i=1}^{n} \sigma_{i}: R \rightarrow \prod_{i=1}^{n} R_{i}$ is an isomorphism.

(c) Let us identify the ring $R$ with $\prod_{i=1}^{n} R_{i}$ via $\sigma$. Then $S_{i}=R_{1} \times \cdots \times R_{i}^{*} \times \cdots \cdots \times R_{n}=$ $\sigma_{i}^{-1}\left(R_{i}^{*}\right)$ where $R_{i}^{*}=\left(R / \operatorname{ass}\left(S_{i}\right)\right)^{*}$ is the group of units of the ring $R_{i}$, and for each $i=1, \ldots, n$, $R_{i}^{*}=\sigma_{i}\left(S_{i}\right)$.

(d) $\operatorname{ass}\left(S_{i}\right)=R_{1} \times \cdots \times 0 \times \cdots \times R_{n}$ for $i=1, \ldots, n$ where 0 is on $i$ 'th place.

Proof. $(\Rightarrow)$ Theorem 2.9.

$(\Leftarrow)$ By $(2)$ and conditions 1 and 2, the map $\sigma$ is a ring monomorphism since

$$
\operatorname{ker}(\sigma)=\bigcap_{i=1}^{n} \operatorname{ass}\left(S_{i}\right)=\mathfrak{l}_{R}=0 .
$$

The ring homomorphism $\sigma_{i}$ yields a monomorphism $R / \operatorname{ass}\left(S_{i}\right) \rightarrow S_{i}^{-1} R=R_{i}$ which is necessarily an isomorphism, by condition 4. Clearly, $\sigma_{i}: R \rightarrow R / \operatorname{ass}\left(S_{i}\right), r \mapsto r+\operatorname{ass}\left(S_{i}\right)$.

Conditions 1 and $2, \bigcap_{i=1}^{n} \operatorname{ass}\left(S_{i}\right)=0$ and $\operatorname{ass}\left(S_{i}\right)+\operatorname{ass}\left(S_{j}\right)=R$ for all $i \neq j$, imply that the map $\sigma$ is an isomorphism, i.e. the ring $R \simeq R_{1} \times \cdots \times R_{n}$ is a direct product of $n$ left localization maximal rings.

By Theorem 2.9] (1), $S_{i}=R_{1} \times \cdots \times R_{i}^{*} \times \cdots \times R_{n}=\sigma_{i}^{-1}\left(R_{i}^{*}\right)$ and $\operatorname{ass}\left(S_{i}\right)=R_{1} \times \cdots \times 0 \times \cdots \times R_{n}$. Then $R_{i}^{*}=\sigma_{i}\left(S_{i}\right)$ since $\sigma_{i}: R \rightarrow R / \operatorname{ass}\left(S_{i}\right), r \mapsto r+\operatorname{ass}\left(S_{i}\right)$. 


\section{Left localizable rings and their characterization}

The aim of this section is to introduce a new class of rings, the class of left localizable rings, consider their properties, and give their characterizations (Theorem 3.5. Theorem 3.8 and Theorem 3.9) provided they have only finitely many maximal left denominator sets. In particular, we prove that a ring with finitely many maximal left denominator sets is a left localizable ring iff it is a semiprime left Goldie ring such that its left quotient ring is a direct product of division rings (Theorem 3.9). For a semiprime left Goldie ring $R$, it is proved that $\max \cdot \operatorname{Den}_{l}(R) \mid<\infty$. Moreover, elements of $\max \operatorname{Den}_{l}(R)$ are found (Corollary 3.13$)$.

Definition. A ring $R$ is called a left localizable ring if the set of left localizable elements $\mathcal{L}_{l}(R)$ is the largest possible, i.e. $\mathcal{L}_{l}(R)=R \backslash\{0\}$; equivalently, for every nonzero element $r \in R$ there is a left denominator set $S=S(r) \in \operatorname{Den}_{l}(R)$ such that $r \in S$; equivalently, for every nonzero element $r \in R$ there is a left denominator set $S^{\prime}=S^{\prime}(r)$ such that the element $\frac{r}{1} \in S^{\prime-1} R$ is a unit of the ring $S^{\prime-1} R$ (Lemma 2.6).

Similarly, right localizable; left and right localizable; and localizable rings are defined, i.e. where the sets $\mathcal{L}_{r}(R) ; \mathcal{L}_{l, r}(R)$; and $\mathcal{L}(R)$ are equal to $R \backslash\{0\}$, respectively. A localizable ring is also called a two-sided localizable ring.

Theorem 3.1 Let $R=\prod_{i=1}^{n} R_{i}$ be a direct product of rings $R_{i}$. The ring $R$ is a left localizable ring iff the rings $R_{i}$ are so.

Proof. The proof is an easy corollary of Theorem 2.8 that states that $\max \cdot \operatorname{Den}_{l}\left(\prod_{i=1}^{n} R_{i}\right)=$ $\coprod_{i=1}^{n} \max \operatorname{Den}_{l}\left(R_{i}\right)$.

$(\Rightarrow)$ Suppose that the ring $R$ is a left localizable ring. We have to show that the rings $R_{i}$ are so. Each ring $R_{i}$ is a subring of $R$. Let $r_{i} \in R_{i}$ be a nonzero element. The ring $R$ is a left localizable ring. So, $r_{i} \in S_{i}$ for some $S_{i} \in \max \operatorname{Den}_{l}(R)$. Then $S_{i} \in \max \cdot \operatorname{Den}_{l}\left(R_{i}\right)$, by Theorem 2.8 .

$(\Leftarrow)$ Suppose that the rings $R_{i}$ are left localizable rings. Let $r=\left(r_{1}, \ldots, r_{n}\right) \in R$ be a nonzero element. Then $0 \neq r_{i} \in R_{i}$ for some $i$. The ring $R_{i}$ is a left localizable ring, and so $r_{i} \in S_{i}$ for

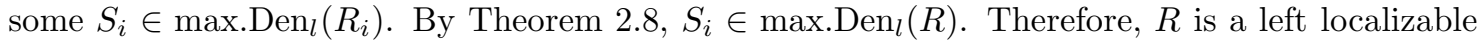
ring.

Lemma 3.2 1. If a ring $R$ is a left (respectively, right; left and right; two-sided) localizable then $\mathfrak{l}_{R}=0$ (respectively, $\left.\operatorname{r} \cdot \operatorname{lrad}(R)=0 ; \operatorname{lr} \cdot \operatorname{lrad}(R)=0 ; \operatorname{lrad}(R)=0\right)$.

2. Let a ring $R=\prod_{i=1}^{n} R_{i}$ be a direct product of left localization maximal rings $R_{i}$. Then $R$ is a left localizable ring iff the rings $R_{i}$ are division rings.

Proof. 1. Statements are easy corollaries of Lemma 2.7

2. Corollary 2.10 $\square$

Properties of the maximal left quotient rings of a ring. The next theorem describes various properties of the maximal left quotient rings of a ring, in particular, their groups of units and their largest left quotient rings.

Theorem 3.3 [2] Let $S \in \max ^{-D^{2}} \operatorname{Den}_{l}(R), A=S^{-1} R, A^{*}$ be the group of units of the ring $A$; $\mathfrak{a}:=\operatorname{ass}(S), \pi_{\mathfrak{a}}: R \rightarrow R / \mathfrak{a}, a \mapsto a+\mathfrak{a}$, and $\sigma_{\mathfrak{a}}: R \rightarrow A, r \mapsto \frac{r}{1}$. Then

1. $S=S_{\mathfrak{a}}(R), S=\pi_{\mathfrak{a}}^{-1}\left(S_{0}(R / \mathfrak{a})\right), \pi_{\mathfrak{a}}(S)=S_{0}(R / \mathfrak{a})$ and $A=S_{0}(R / \mathfrak{a})^{-1} R / \mathfrak{a}=Q_{l}(R / \mathfrak{a})$.

2. $S_{0}(A)=A^{*}$ and $S_{0}(A) \cap(R / \mathfrak{a})=S_{0}(R / \mathfrak{a})$.

3. $S=\sigma_{\mathfrak{a}}^{-1}\left(A^{*}\right)$.

4. $A^{*}=\left\langle\pi_{\mathfrak{a}}(S), \pi_{\mathfrak{a}}(S)^{-1}\right\rangle$, i.e. the group of units of the ring $A$ is generated by the sets $\pi_{\mathfrak{a}}(S)$ and $\pi_{\mathfrak{a}}^{-1}(S):=\left\{\pi_{\mathfrak{a}}(s)^{-1} \mid s \in S\right\}$. 
5. $A^{*}=\left\{\pi_{\mathfrak{a}}(s)^{-1} \pi_{\mathfrak{a}}(t) \mid s, t \in S\right\}$.

6. $Q_{l}(A)=A$ and $\operatorname{Ass}_{l}(A)=\{0\}$. In particular, if $T \in \operatorname{Den}_{l}(A, 0)$ then $T \subseteq A^{*}$.

Lemma 3.4 Let $R$ be a ring and $S \in \max ^{-D} \operatorname{Den}_{l}(R)$. Then the ring $S^{-1} R$ is a division ring iff $R=S \cup \operatorname{ass}(S)$.

Remark. For any left Ore set $T, T \cap \operatorname{ass}(T)=\emptyset$.

Proof. Let $\sigma: R \rightarrow S^{-1} R, r \mapsto \frac{r}{1}$, and $\left(S^{-1} R\right)^{*}$ be the group of units of the ring $S^{-1} R$. By Theorem 3.3. (3), $S=\sigma^{-1}\left(\left(S^{-1} R\right)^{*}\right)$ for all $S \in \max ^{2} \operatorname{Den}_{l}(R)$.

$(\Rightarrow)$ Let $s \in R$. Suppose that $s \notin \operatorname{ass}(S)$, we have to show that $s \in S$. Clearly, $\frac{s}{1} \neq 0$ in $S^{-1} R$. Therefore, $\frac{s}{1} \in\left(S^{-1} R\right)^{*}$ since $S^{-1} R$ is a division ring. Then $s \in \sigma^{-1}\left(\left(S^{-1} R\right)^{*}\right)=S$.

$(\Leftarrow)$ If $R=S \cup \operatorname{ass}(S)$ then this union is a disjoint union since $S \cap \operatorname{ass}(S)=\emptyset$. In particular, $S+\operatorname{ass}(S) \subseteq S$. Let $\frac{r}{1}$ (where $r \in R$ ) be a nonzero element of the ring $S^{-1} R$. Then $r \notin \operatorname{ass}(S)$, and so $r \in S$, i.e. $\frac{r}{1}$ is a unit in the ring $S^{-1} R$. Therefore, the ring $S^{-1} R$ is a division ring.

Characterization of left localizable rings. The next theorem gives a characterization of left localizable rings with finite number of maximal left denominator sets.

Theorem 3.5 Let $R$ be a ring with $\max \operatorname{Den}_{l}(R)=\left\{S_{1}, \ldots, S_{n}\right\}$. Let $\mathfrak{a}_{i}:=\operatorname{ass}\left(S_{i}\right) ; \sigma_{i}: R \rightarrow$ $R_{i}:=S_{i}^{-1} R, r \mapsto \frac{r}{1}=r_{i}$; and $\sigma:=\prod_{i=1}^{n} \sigma_{i}: R \rightarrow \prod_{i=1}^{n} R_{i}, r \mapsto\left(r_{1}, \ldots, r_{n}\right)$. The following statements are equivalent.

1. The ring $R$ is a left localizable ring.

2. $\mathfrak{l}_{R}=0$ and the rings $R_{1}, \ldots, R_{n}$ are division rings.

3. The homomorphism $\sigma$ is an injection and the rings $R_{1}, \ldots, R_{n}$ are division rings.

Proof. The rings $R_{i}$ are left localization maximal (Theorem 3.3.(6)).

$(2 \Leftrightarrow 3)$ Statements 2 and 3 are equivalent since $\operatorname{ker}(\sigma)=\bigcap_{i=1}^{n} \operatorname{ass}\left(S_{i}\right)=\mathfrak{l}_{R}$.

$(1 \Leftarrow 2)$ Since $\mathfrak{l}_{R}=0$, the map $\sigma$ (see (2) ) is a ring monomorphism. So, every element $r$ is a unique $n$-tuple $\left(r_{1}, \ldots, r_{n}\right)$ where $r_{i} \in R_{i}$. The rings $R_{1}, \ldots, R_{n}$ are division rings. Let $r=\left(r_{i}\right) \in R$ be a nonzero element of $R$, then $r_{i} \neq 0$ for some $i$, and so $\sigma_{i}(r)=r_{i} \in R_{i}^{*}$. Therefore, every nonzero element of the ring is left localizable.

$(1 \Rightarrow 2)$ Suppose that every nonzero element of the ring $R$ is left localizable, i.e. $\mathcal{N} \mathcal{L}_{l}(R)=\{0\}$. By Lemma 2.7 (2), $\mathfrak{l}_{R}=0$, and so, by (2), the map $\sigma$ is a ring monomorphism. We identify the ring $R$ with its image $\sigma(R)$ in the ring $\prod_{i=1}^{n} R_{i}$, i.e. $r=\left(r_{1}, \ldots, r_{n}\right)$ where $r_{i}=\sigma_{i}(r)$.

If $n=1$ then $\sigma_{1}: R \rightarrow S_{1}^{-1} R, r \mapsto \frac{r}{1}$, is a monomorphism, and so $\operatorname{ass}\left(S_{1}\right)=0$. By the assumption $S_{1}=\mathcal{L}_{l}(R)=R \backslash\{0\}$, i.e. $S_{1}^{-1} R$ is a division ring.

Let $n \geq 2$. By Proposition 2.4 the ideals $\mathfrak{a}_{i}:=\operatorname{ass}\left(S_{i}\right), i=1, \ldots, n$, are non-comparable, i.e.

$$
\mathfrak{a}_{i} \not \subseteq \mathfrak{a}_{j}, \quad i \neq j .
$$

In particular, $\mathfrak{a}_{i} \neq 0$ for all $i$. The proof consists of several steps.

Step 1: For all $i$,

$$
S_{i} \cap \bigcap_{j \neq i} \mathfrak{a}_{j} \neq \emptyset .
$$

Fix $r \in S_{i}$. In particular, $r=\left(r_{1}, \ldots, r_{n}\right)$ with $r_{i} \in R_{i}^{*}$, and vice versa, i.e. if $r_{i} \in R_{i}^{*}$ then $r \in \sigma_{i}^{-1}\left(R_{i}^{*}\right)=S_{i}$. Fix a nonzero element, say $r \in S_{i}$, such that the $n$-tuple $r=\left(r_{1}, \ldots, r_{n}\right)$ has the largest possible number of zeros, say $m=n-s$ for some $s$. We claim that $s=1$. Up to order, we may assume that $i=1$,

$$
r=\left(r_{1}, \ldots, r_{s}, 0, \ldots, 0\right)
$$

and $r_{j} \neq 0$ for $j=1, \ldots, s$, and $r_{1} \in R_{1}^{*}$. Suppose that $s \neq 1$, we seek a contradiction. Then, for all elements $a_{s} \in \mathfrak{a}_{s}$

$$
\sigma\left(a_{s}\right) \sigma(r)=\left(\sigma_{1}\left(a_{s}\right) r_{1}, \ldots, \sigma_{s-1}\left(a_{s}\right) r_{s-1}, 0, \ldots, 0\right) .
$$


By the maximality of $m$ we must have $\sigma\left(a_{s}\right) \sigma(r)=0$. In particular, $\sigma_{1}\left(a_{s}\right) r_{1}=0$, and so $\sigma_{1}\left(a_{s}\right)=0$ since $r_{1} \in R^{*}$, i.e. $\mathfrak{a}_{s} \subseteq \mathfrak{a}_{1}$, a contradiction, (Proposition 2.4). Therefore, $s=1$, and $\sigma(r) \in S_{1} \cap \bigcap_{j \neq 1} \mathfrak{a}_{j} \neq 0$. The proof of Step 1 is complete.

Step 2: For each $i$, the ring $R_{i}$ is a division ring.

Without loss of generality we may assume that $i=1$, then $S_{1} \cap \bigcap_{j=2}^{n} \mathfrak{a}_{j} \neq \emptyset$ (Step 1). Fix an element $s \in S_{1} \cap \bigcap_{j=2}^{n} \mathfrak{a}_{j}$. Then $\sigma(s)=\left(s_{1}, 0, \ldots, 0\right)$ and $s_{1} \in R_{1}^{*}$ (since $s \in S_{1}$ and $R_{1}=S_{1}^{-1} R$ ). Suppose that the ring $R_{1}$ is not a division ring, we seek a contradiction. Then, by Lemma 3.4. $R_{1} \neq S_{1} \cup \mathfrak{a}_{1}$. Fix an element $r \in R \backslash\left(S_{1} \cup \mathfrak{a}_{1}\right)$. Then $\sigma(r)=\left(r_{1}, \ldots, r_{n}\right)$ with $S_{1}^{-1} R \ni r_{1} \neq 0$ (since $r \notin \mathfrak{a}_{1}$ ). Then

$$
\sigma(s r)=\left(s_{1} r_{1}, 0, \ldots, 0\right) \in \prod_{i=1}^{n} R_{i}
$$

with $s_{1} r_{1} \neq 0$ since $s_{1} \in R_{1}^{*}$ and $r_{1} \neq 0$. Therefore, $s r \notin \bigcup_{j=2}^{n} S_{j}$ (since $s r \in \cap_{j=2}^{n} \mathfrak{a}_{j}$, by the choice of $s$ ). Then necessarily $s r \in S_{1}$ since $s r \in R \backslash\{0\}=\bigcup_{i=1}^{n} S_{i}$ (the ring $R$ is a left localizable ring). Then $\sigma_{1}(r)=\sigma_{1}(s)^{-1} \sigma_{1}(s r) \in R_{1}^{*}$, and so $r \in \sigma_{1}^{-1}\left(R_{1}^{*}\right)=S_{1}$, by Theorem 3.3. (3). This contradicts to the fact that $r \in R \backslash\left(S_{1} \cup \mathfrak{a}_{1}\right)$. Therefore, $R_{1}$ is a division ring. The proof of Step 2 and the theorem is complete.

Corollary 3.6 Let $R$ be a left localizable ring with $\max \operatorname{Den}_{l}(R)=\left\{S_{1}, \ldots, S_{n}\right\}$ where $n \geq 2$. Then, for all $i=1, \ldots, n, S_{i} \cap \bigcap_{j \neq i} \mathfrak{a}_{j}=\left(\bigcap_{j \neq i} \mathfrak{a}_{j}\right) \backslash\{0\} \neq \emptyset$ where $\mathfrak{a}_{i}:=\operatorname{ass}\left(S_{i}\right)$.

Proof. By Step 1 of the proof of Theorem 3.5 $S_{i} \cap \bigcap_{j \neq i} \mathfrak{a}_{j} \neq \emptyset$. The equality in the corollary follows from the fact that $\bigcap_{i=1}^{n} \mathfrak{a}_{j}=0$ and $R_{i}=S_{i} \cup \mathfrak{a}_{i}$ is a disjoint union (Lemma 3.4, since $R_{i}$ is a division ring, by Theorem 3.5). Then $\left(\bigcap_{j \neq i} \mathfrak{a}_{j}\right) \backslash\{0\} \neq \emptyset$.

Proposition 3.7 Let $R$ be a left localizable ring with $\max \operatorname{Den}_{l}(R)=\left\{S_{1}, \ldots, S_{n}\right\}$ where $n \geq 2$. We keep the notation of Theorem 3.5. Let $C_{i}^{\prime}:=\left(\bigcap_{j \neq i} \mathfrak{a}_{j}\right) \backslash\{0\}$. Then

1. $C^{\prime}:=C_{1}^{\prime}+\cdots+C_{n}^{\prime} \in \operatorname{Den}_{l}(R, 0)$ and $C^{\prime-1} R \simeq R_{1} \times \cdots \times R_{n}$.

2. For $i=1, \ldots, n, C_{i}^{\prime} \in \operatorname{Den}_{l}\left(R, \mathfrak{a}_{i}\right)$ and $C_{i}^{\prime-1} R=R_{i}$.

Proof. Clearly, all $C_{i}^{\prime} \neq \emptyset$ (Corollary [3.6); $C_{i}^{\prime} C_{j}^{\prime}=0$ for all $i \neq j$ (since $C_{i}^{\prime} C_{j}^{\prime} \subseteq \bigcap_{k=1}^{n} \mathfrak{a}_{k}=0$, Theorem 3.5), and $\sigma\left(C_{i}^{\prime}\right) \subseteq 0 \times \cdots \times 0 \times R_{i}^{*} \times 0 \times \cdots \times 0$ since $C_{i}^{\prime}=S_{i} \cap \bigcap_{j \neq i} \mathfrak{a}_{j}$ (Corollary 3.6). Therefore, the set $C^{\prime}$ consists of regular elements of the ring $R$ (since the map $\sigma: R \rightarrow \prod_{i=1}^{n} R_{i}$ is a monomorphism) and is obviously multiplicatively closed. Let us identify the set $C_{i}^{\prime}$ with its image in the division ring $R_{i}$ via $\sigma$. Then $\operatorname{ass}\left(C_{i}^{\prime}\right)=\mathfrak{a}_{i}$ (since $\mathcal{C}_{i}^{\prime} S_{i} \subseteq \mathcal{C}_{i}^{\prime}$ ). Notice that $C_{i}^{\prime} \cup\{0\}=\bigcap_{j \neq i} \mathfrak{a}_{j}$ is an ideal of the ring $R$. Fix an element $c_{i} \in C_{i}^{\prime}$, then each element $s^{-1} r \in R_{i}$, where $s \in S_{i}$ and $r \in R$, can be written as a left fraction

$$
s^{-1} r=s^{-1} c_{i}^{-1} c_{i} r=\left(c_{i} s\right)^{-1} c_{i} r, \text { where } c_{i} s \in C_{i}^{\prime} \text { and } c_{i} r \in C_{i}^{\prime} \cup\{0\} .
$$

Therefore, $C_{i}^{\prime} \in \operatorname{Den}_{l}\left(R, \mathfrak{a}_{i}\right)$ and $C_{i}^{\prime-1} R=R_{i}$, and statement 2 holds. So, any element $\alpha$ of the ring $\prod_{i=1}^{n} R_{i}$ can be written as $\alpha=\left(c_{1}^{-1} r_{1}, \ldots c_{n}^{-1} r_{n}\right)$ where $c_{i} \in C_{i}^{\prime}$ and $r_{i} \in C_{i}^{\prime} \cup\{0\}$, and so

$$
\alpha=\left(c_{1}^{-1} r_{1}, \ldots c_{n}^{-1} r_{n}\right)=\left(c_{1}+\cdots+c_{n}\right)^{-1}\left(r_{1}+\cdots+r_{n}\right)
$$

where $c:=c_{1}+\cdots+c_{n} \in C^{\prime}$ and $r:=r_{1}+\cdots+r_{n} \in\left(\sum_{i=1}^{n} C_{i}^{\prime}\right) \cup\{0\} \subseteq R$. So, every element $\alpha$ of the ring $\prod_{i=1}^{n} R_{i}$ can be written as $\alpha=c^{-1} r$ for some elements $c \in C^{\prime}$ and $r \in R$. Since the set $C^{\prime}$ consists of regular elements, the fact above $\left(\alpha=c^{-1} r\right)$ is equivalent to the fact that $C^{\prime}$ is a left Ore set, and so $C^{\prime} \in \operatorname{Den}_{l}(R, 0)$. Clearly, $C^{\prime-1} R=R_{1} \times \cdots \times R_{n}$.

The sets $C_{i}^{\prime}$ in Theorem 3.7 have remarkable properties: $C_{i}^{\prime} \subseteq S_{i}, C_{i}^{\prime-1} R=S_{i}^{-1} R$ and $\operatorname{ass}\left(c_{i}^{\prime} \cdot\right)=$ $\operatorname{ass}\left(S_{i}\right)$ for all elements $c_{i}^{\prime} \in C_{i}^{\prime}$ where $c_{i}^{\prime} \cdot: R \rightarrow R, r \mapsto c_{i}^{\prime} r$ (Theorem 4.5). In fact, the set $C_{i}^{\prime}$ is the core of $S_{i}$ (Theorem 4.5).

The next theorem is a useful criterion for a ring $R$ to be a left localizable ring with $\left|\max \operatorname{Den}_{l}(R)\right|<$ $\infty$. 
Theorem 3.8 Let $R$ be a ring. The following statements are equivalent.

1. The ring $R$ is a left localizable ring with $\left|\max \operatorname{Den}_{l}(R)\right|<\infty$.

2. There are left denominator sets $S_{1}^{\prime}, \ldots, S_{n}^{\prime} \in \operatorname{Den}_{l}(R)$ such that the rings $R_{i}:=S_{i}^{\prime-1} R$, $i=1, \ldots, n$, are division rings and the map

$$
\sigma:=\prod_{i=1}^{n} \sigma_{i}: R \rightarrow \prod_{i=1}^{n} R_{i}, \quad r \mapsto\left(\sigma_{1}(r), \ldots, \sigma_{n}(r)\right)
$$

is an injection where $\sigma_{i}: R \rightarrow R_{i}, r \mapsto \frac{r}{1}$.

If one of the equivalent conditions holds and none of the rings $R_{i}$ can be dropped with preserving the injectivity of $\sigma$ then $n=\left|\max _{\operatorname{Den}}(R)\right|$, and $\max \cdot \operatorname{Den}_{l}(R)=\left\{S_{1}, \ldots, S_{n}\right\}$ where $S_{i}=\sigma_{i}^{-1}\left(R_{i}^{*}\right)$.

Proof. $(1 \Rightarrow 2)$ Theorem 3.5 .

$(2 \Leftarrow 1)$ Suppose that statement 2 holds and we assume that none of the rings $R_{i}$ can be dropped, i.e. for each $i=1, \ldots, n, \bigcap_{j \neq i} \mathfrak{a}_{i} \neq 0$ where $\mathfrak{a}_{i}:=\operatorname{ass}\left(S_{i}^{\prime}\right)$. We are going to prove that the implication $(2 \Leftarrow 1)$ holds and that $\max \cdot \operatorname{Den}_{l}(R)=\left\{S_{1}, \ldots, S_{n}\right\}$.

Every division ring is a left localization maximal ring. By (Theorem 3.12.(3), 2]), $S_{i} \in$ $\max _{\operatorname{Den}}(R)$. Clearly, $S_{i}^{\prime} \subseteq \sigma_{i}^{-1}\left(R_{i}^{*}\right)=S_{i}$ and the natural ring homomorphism $R_{i}=S_{i}^{\prime-1} R \rightarrow$ $S_{i}^{-1} R, \frac{r}{1} \rightarrow \frac{r}{1}$, is an isomorphism, and so $\mathfrak{a}_{i}=\operatorname{ass}\left(S_{i}\right)$. We identify the rings $R_{i}$ and $S_{i}^{-1} R$ via the isomorphism above. Since $S_{i} \in \max \operatorname{Den}_{l}(R)$ and $S_{i}^{-1} R$ is a division ring, we see that (by Lemma 3.4) $R=S_{i} \amalg \mathfrak{a}_{i}$, a disjoint union. Then

$$
S_{i} \cap \bigcap_{j \neq i} \mathfrak{a}_{j}=\left(\bigcap_{j \neq i} \mathfrak{a}_{j}\right) \backslash\{0\} \neq \emptyset
$$

since $\bigcap_{i=1}^{n} \mathfrak{a}_{i}=\operatorname{ker}(\sigma)=0$. This fact together with the fact that $R=S_{i} \amalg \mathfrak{a}_{i}$ for $i=1, \ldots, n$, imply that the sets $S_{1}, \ldots, S_{n}$ are distinct.

Finally, we claim that $\max \cdot \operatorname{Den}_{l}(R)=\left\{S_{1}, \ldots, S_{n}\right\}$. Suppose that this is not true, that is there exists a set $S \in \max . \operatorname{Den}_{l}(R)$ distinct from the sets $S_{1}, \ldots, S_{n}$, we seek a contradiction. For each element $i=1, \ldots, n$, by using the disjoint union $R=S_{i} \amalg \mathfrak{a}_{i}$ and the fact that $S \neq S_{i}$, we can find an element, say $t_{i}$ such that $t_{i} \in S \cap \mathfrak{a}_{i}$ (notice that $S \nsubseteq S_{i}$ since $S, S_{i} \in \max \operatorname{Den}_{l}(R)$ ). Then $\sigma_{i}\left(t_{i}\right)=0$, and so $\sigma(t)=0$ where $t:=t_{1}, \cdots t_{n} \in S$. On the other hand, $\sigma(t) \neq 0$ since $t \in S$, a contradiction.

A left localizable ring $R$ with $\left|\max _{\text {Den }}(R)\right|<\infty$ is a semiprime left Goldie ring such that $Q_{l, c l}(R)$ is a direct product of division rings, and vice versa.

Theorem 3.9 Let $R$ be a ring. The following statements are equivalent.

1. The ring $R$ is a left localizable ring with $n:=\left|\max _{\operatorname{Den}}(R)\right|<\infty$.

2. $Q_{l, c l}(R)=R_{1} \times \cdots \times R_{n}$ where $R_{i}$ are division rings.

3. The ring $R$ is a semiprime left Goldie ring with $\operatorname{udim}(R)=|\operatorname{Min}(R)|=n$ where $\operatorname{Min}(R)$ is the set of minimal prime ideals of the ring $R$.

4. $Q_{l}(R)=R_{1} \times \cdots \times R_{n}$ where $R_{i}$ are division rings.

Proof. ( $2 \Leftrightarrow 3)$ It is known and easy to prove (use Goldie's Theorem).

$(1 \Rightarrow 2)$ By Proposition 3.7, $C^{\prime-1} R=R_{1} \times \cdots \times R_{n}$ where $R_{i}$ are division rings. In particular, $C^{\prime-1} R$ is a semisimple (Artinian) ring. Clearly, $C^{\prime} \subseteq S_{0}(R)$ where $S_{0}(R)$ is the largest regular left Ore set of the ring $R$. Then

$$
Q_{l}(R)=S_{0}(R)^{-1} R=Q_{l}\left(C^{\prime-1} R\right)=Q_{l}\left(R_{1} \times \cdots \times R_{n}\right)=R_{1} \times \cdots \times R_{n} .
$$

Since $Q_{l}(R)$ is a semisimple ring, $Q_{l, c l}(R)=Q_{l}(R)$, by (Corollary 2.10, 2] ). 
$(2 \Rightarrow 1)$ The map $\sigma: R \rightarrow Q_{l, c l}(R)=R_{1} \times \cdots \times R_{n}, r \mapsto \frac{r}{1}$, is a monomorphism. Any division ring is a left localization maximal ring. By Proposition 3.10 $(4), \max ^{-D^{2} n_{l}}(R)=\left\{T_{1}, \ldots, T_{n}\right\}$ where $T_{i}=\sigma^{-1}\left(R_{1} \times \cdots \times R_{i}^{*} \times \cdots \times R_{n}\right)$ and $R_{i} \simeq T_{i}^{-1} R$. Since $R_{1}, \ldots, R_{n}$ are division rings and $\sigma=\prod_{i=1}^{n} \sigma_{i}: R \rightarrow \prod_{i=1}^{n} T_{i}^{-1} R, r \mapsto\left(\sigma_{1}(r), \ldots, \sigma_{n}(r)\right)$, is a monomorphism where $\sigma_{i}: R \rightarrow T_{i}^{-1} R, r \mapsto \frac{r}{1}$, the ring $R$ is a left localizable ring (Theorem 3.5).

$(3 \Leftrightarrow 4)$ This equivalence is a particular case of a more general result (Corollary 2.10, [2]): Let $R$ be a ring. Then $Q_{l}(R)$ is a semisimple ring iff $Q_{l, c l}(R)$ is a semisimple ring, and in this case $Q_{l}(R)=Q_{l, c l}(R)$.

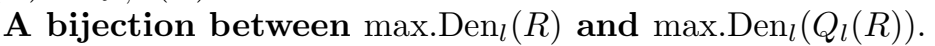

Proposition 3.10 [3] Let $R$ be a ring, $S_{l}$ be the largest regular left Ore set of the ring $R, Q_{l}:=$ $S_{l}^{-1} R$ be the largest left quotient ring of the ring $R$, and $\mathcal{C}$ be the set of regular elements of the ring $R$. Then

1. $S_{l} \subseteq S$ for all $S \in \max \operatorname{Den}_{l}(R)$. In particular, $\mathcal{C} \subseteq S$ for all $S \in \max ^{-D_{e n}}(R)$ provided $\mathcal{C}$ is a left Ore set.

2. Either $\max \cdot \operatorname{Den}_{l}(R)=\{\mathcal{C}\}$ or, otherwise, $\mathcal{C} \notin \max \cdot \operatorname{Den}_{l}(R)$.

3. The map

$$
\max _{\operatorname{Den}}(R) \rightarrow \max \cdot \operatorname{Den}_{l}\left(Q_{l}\right), \quad S \mapsto S Q_{l}^{*}=\left\{c^{-1} s \mid c \in S_{l}, s \in S\right\},
$$

is a bijection with the inverse $\mathcal{T} \mapsto \sigma^{-1}(\mathcal{T})$ where $\sigma: R \rightarrow Q_{l}, r \mapsto \frac{r}{1}$, and $S Q_{l}^{*}$ is the sub-semigroup of $\left(Q_{l}, \cdot\right)$ generated by the set $S$ and the group $Q_{l}^{*}$ of units of the ring $Q_{l}$, and $S^{-1} R=\left(S Q_{l}^{*}\right)^{-1} Q_{l}$.

4. If $\mathcal{C}$ is a left Ore set then the map

$$
\max \cdot \operatorname{Den}_{l}(R) \rightarrow \max \cdot \operatorname{Den}_{l}(Q), \quad S \mapsto S Q^{*}=\left\{c^{-1} s \mid c \in \mathcal{C}, s \in S\right\},
$$

is a bijection with the inverse $\mathcal{T} \mapsto \sigma^{-1}(\mathcal{T})$ where $\sigma: R \rightarrow Q, r \mapsto \frac{r}{1}$, and $S Q^{*}$ is the sub-semigroup of $(Q, \cdot)$ generated by the set $S$ and the group $Q^{*}$ of units of the ring $Q$, and $S^{-1} R=\left(S Q^{*}\right)^{-1} Q$.

Theorem 3.11 Let $R$ be a ring, $T \in \operatorname{Den}_{l}(R, 0)$ and $\sigma: R \rightarrow T^{-1} R, r \mapsto \frac{r}{1}$. Then

1. $T \subseteq S$ for all $S \in \max \cdot \operatorname{Den}_{l}(R)$.

2. The map

$$
\max _{\operatorname{Den}}(R) \rightarrow \max \cdot \operatorname{Den}_{l}\left(T^{-1} R\right), \quad S \mapsto \widetilde{S},
$$

is a bijection with the inverse $\mathcal{T} \mapsto \sigma^{-1}(\mathcal{T})$ where $\widetilde{S}$ is the multiplicative monoid generated in the ring $T^{-1} R$ by $\sigma(S)$ and $\sigma(T)^{-1}:=\left\{t^{-1} \mid t \in T\right\}$ and $S^{-1} R \simeq \widetilde{S}^{-1}\left(T^{-1} R\right)$, and $S^{-1} R \simeq \widetilde{S}^{-1}\left(T^{-1} R\right)$.

Proof. 1. Lemma 2.2,

2. Statement 2 follows from statement 1 and Proposition 3.4.(1), [2].

Theorem 3.12 Let $R$ be a ring, $T \in \operatorname{Den}_{l}(R, 0)$ be such that $T^{-1} R=R_{1} \times \cdots \times R_{n}$ is a direct product of left localization maximal rings; and $\tau: R \rightarrow T^{-1} R, r \mapsto \frac{r}{1}=\left(r_{1}, \ldots, r_{n}\right)$. Then $\max _{\operatorname{Den}}(R)=\left\{T_{1}, \ldots, T_{n}\right\}$ where $T_{i}=\tau^{-1}\left(S_{i}\right), S_{i}:=R_{1} \times \cdots \times R_{i}^{*} \times \cdots \times R_{n}$ and $T_{i}^{-1} R \simeq R_{i}$.

Proof. Proposition follows from Theorem 3.11 and Theorem 2.9 (1).

The next theorem shows that $\left|\max \cdot \operatorname{Den}_{l}(R)\right|<\infty$ for all semiprime left Goldie rings. 
Corollary 3.13 Let $R$ be a semiprime left Goldie ring, $\mathcal{C}$ be the set of regular element of the ring $R, Q_{l, c l}=\prod_{i=1}^{n} Q_{i}$ where $Q_{i}$ are simple Artinian rings (by Goldie's Theorem), $\sigma: R \rightarrow Q_{l, c l}$, $r \mapsto \frac{r}{1}$. Then

1. $C \subseteq S$ for all $S \in \max \cdot \operatorname{Den}_{l}(R)$.

2. $\max \cdot \operatorname{Den}_{l}(R)=\left\{S_{1}, \ldots, S_{n}\right\}$, $\max _{i} \cdot \operatorname{Den}_{l}\left(Q_{l, c l}(R)\right)=\left\{S_{1}^{\prime}, \ldots, S_{n}^{\prime}\right\}$ where $S_{i}=\sigma^{-1}\left(S_{i}^{\prime}\right)$ and $S_{i}^{\prime}:=Q_{1} \times \cdots \times Q_{i}^{*} \times \cdots \times Q_{n}$ for $i=1, \ldots, n$. The map

$$
\max _{\operatorname{Den}}(R) \rightarrow \max _{l} \operatorname{Den}_{l}\left(Q_{l, c l}(R)\right), \quad S \mapsto \widetilde{S},
$$

is a bijection with inverse $\mathcal{T} \mapsto \sigma^{-1}(\mathcal{T})$ where $\widetilde{S}$ is the multiplicative monoid generated in $Q_{l, c l}(R)$ by $\sigma(S)$ and $\sigma(\mathcal{C})^{-1}=\left\{c^{-1} \mid c \in \mathcal{C}\right\}$.

3. $S_{i}^{-1} R \simeq Q_{i}$ for $i=1, \ldots, n$.

Proof. This is a particular case of Theorem 3.11 where $T=C$.

\section{The core of a left Ore set}

The aim of this section is to establish several properties of the core $S_{c}$ of a left Ore set $S$ of a ring $R$, and when it exists (for example for a right Noetherian ring $R$ ) it is a powerful tool in studying localizations (Theorem 4.2). A characterization of the core is given (Proposition 4.4). The core shares remarkable properties (Lemma 4.1. Theorem 4.2).

Definition, [3] Let $R$ be a ring and $S$ be its left Ore set. The subset of $S$,

$$
S_{c}:=\{s \in S \mid \operatorname{ker}(s \cdot)=\operatorname{ass}(S)\}
$$

is called the core of the left Ore set $S$ where $s \cdot R \rightarrow R, r \mapsto s r$.

If $S_{c} \neq \emptyset$ then $S_{c} S_{c} \subseteq S_{c}$, i.e. the core $S_{c}$ is a multiplicative set. If $\operatorname{ass}(S)=0$ then $S_{c}=S$.

Lemma 4.1 If $S \in \operatorname{Den}_{l}(R)$ and $S_{c} \neq \emptyset$ then

1. $S S_{c} \subseteq S_{c}$.

2. For any $s \in S$ there exists an element $t \in S$ such that $t s \in S_{c}$.

Proof. 1. Trivial.

2. Statement 2 follows directly from the left Ore condition: fix an element $s_{c} \in S_{c}$, then $t s=r s_{c} \in S$ for some elements $t \in S$ and $r \in R$. Since $\operatorname{ass}(S) \supseteq \operatorname{ker}(t s \cdot)=\operatorname{ker}\left(r s_{c} \cdot\right) \supseteq \operatorname{ker}\left(s_{c} \cdot\right)=$ $\operatorname{ass}(S)$, i.e. $\operatorname{ker}(t s \cdot)=\operatorname{ass}(S)$, we have $t s \in S_{c}$.

Theorem 4.2 Suppose that $S \in \operatorname{Den}_{l}(R, \mathfrak{a})$ and $S_{c} \neq \emptyset$. Then

1. $S_{c} \in \operatorname{Den}_{l}(R, \mathfrak{a})$.

2. The map $\theta: S_{c}^{-1} R \rightarrow S^{-1} R, s^{-1} r \mapsto s^{-1} r$, is a ring $R$-isomorphism. $S o, S_{c}^{-1} R \simeq S^{-1} R$.

Proof. 1. By Lemma 4.1(1), $S_{c} S_{c} \subseteq S_{c}$, that is the set $S_{c}$ is a multiplicative set. By Lemma 4.1. (2), $S_{c} \in \operatorname{Ore}_{l}(R)$ : for any elements $s_{c} \in S_{c}$ and $r \in R$, there are elements $s \in S$ and $r^{\prime} \in R$ such that $s r=r^{\prime} s_{c}$ (since $S \in \mathrm{Ore}_{l}(R)$ ). By Lemma 4.1.(2), $s_{c}^{\prime}:=t s \in S_{c}$ for some $t \in S$. Then $s_{c}^{\prime} r=t r^{\prime} s_{c}$.

If $r s_{c}=0$ for some elements $r \in R$ and $s_{c} \in S_{c}$ then $s r=0$ for some element $s \in S$ (since $S \in \operatorname{Den}_{l}(R)$ ). By Lemma 4.1.(2), $s_{c}^{\prime}:=t s \in S_{c}$ for some element $t \in S$, hence $s_{c}^{\prime} r=0$. Therefore, $S_{c} \in \operatorname{Den}_{l}(R, \mathfrak{a})$. 
2. By statement 1 and the universal property of left Ore localization, the map $\theta$ is a well-defined monomorphism. By Lemma 4.1.(2), $\theta$ is also a surjection: let $s^{-1} r \in S^{-1} R$, and $s_{c}:=t s \in S_{c}$ for some element $t \in S$ (Lemma 4.1(2)). Then

$$
s^{-1} r=s^{-1} t^{-1} \operatorname{tr}=(t s)^{-1} t r=s_{c}^{-1} t r .
$$

Let $S$ be a left Ore set of a ring $R$. The set of right annihilators $\operatorname{ker}(S \cdot):=\{\operatorname{ker}(s \cdot) \mid s \in S\}$ is a poset with respect to $\subseteq$. Let

$$
\max (S):=\{s \in S \mid \operatorname{ker}(s \cdot) \text { is a maximal element of } \operatorname{ker}(S \cdot)\} .
$$

The set $\max (S)$ is a non-empty set for a ring with ACC on right annihilators (for example, when $R$ is a right Noetherian ring).

Lemma 4.3 Let $S \in \operatorname{Ore}_{l}(R)$. Then $\operatorname{ass}(S)=\bigcup_{s \in S} \operatorname{ker}(s \cdot)=\sum_{s \in S} \operatorname{ker}(s \cdot)$.

Proof. Let $U$ and $\Sigma$ stand for the union and the sum respectively. Then $\operatorname{ass}(S) \supseteq \Sigma \supseteq U=$ $\operatorname{ass}(S)$, and so $\operatorname{ass}(S)=\Sigma=U$.

Proposition 4.4 Let $S$ be a left Ore set of a ring $R$. Then $S_{c}=\max (S)$.

Proof. If $S_{c} \neq \emptyset$ then $S_{c}=\max (S)$, by Lemma 4.3 ,

Suppose that $S_{c}=\emptyset$. To finish the proof we have to show that $\max (S)=\emptyset$. Suppose that this is not the case, we seek a contradiction. Let $\mathfrak{a}=\operatorname{ass}(S)$. Fix $s \in \max (S)$. Then $\mathfrak{b}:=\operatorname{ker}(s \cdot) \subseteq \mathfrak{a}$. We claim that $\mathfrak{b}=\mathfrak{a}$, i.e. $s \in S_{c}$, a contradiction. To prove the claim we have to show that for any element $t \in S, \operatorname{ker}(t \cdot) \subseteq \mathfrak{b}$ (see Lemma 4.3). Notice that $\operatorname{ker}\left(s^{\prime} s \cdot\right)=\mathfrak{b}$ for all $s^{\prime} \in S$, by the maximality of $\operatorname{ker}(s \cdot)$. Using the left Ore condition for $S$, we have the equality $s_{1} s=r t$ for some elements $s_{1} \in S$ and $r \in R$. Hence,

$$
\mathfrak{b}=\operatorname{ker}\left(s_{1} s \cdot\right)=\operatorname{ker}(r t \cdot) \supseteq \operatorname{ker}(t \cdot),
$$

as required.

The next theorem gives an explicit description of the cores of maximal left denominator sets

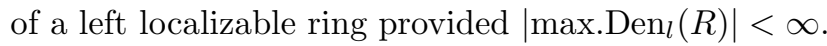

Theorem 4.5 Let $R$ be a left localizable ring such that $\max ^{-D} \operatorname{Den}_{l}(R)=\left\{S_{1}, \ldots, S_{n}\right\}$.

1. If $n=1$ then $S_{1, c}=S_{1}=R \backslash\{0\}$.

2. If $n \geq 2$ then $S_{i, c}=S_{i} \cap \bigcap_{j \neq i} \mathfrak{a}_{i}$ where $\mathfrak{a}_{j}=\operatorname{ass}\left(S_{j}\right)$.

Proof. We keep the notation of Theorem 3.5 and its proof.

1. If $n=1$ then $R$ is a domain $S_{1}=R \backslash\{0\}$ (Theorem 3.5), and so $S_{1, c}=S_{1}=R \backslash\{0\}$.

2. Suppose that $n \geq 2$. For each $i=1, \ldots, n, C_{i}^{\prime}:=S_{i} \cap \bigcap_{j \neq i} \mathfrak{a}_{j} \neq \emptyset$, by Corollary 3.6. By Proposition 3.7. (2), $C_{i}^{\prime} \subseteq S_{i, c}$. By Theorem 3.5, the map

$$
\sigma:=\prod_{i=1}^{n} \sigma_{i}: R \rightarrow \prod_{i=1}^{n} R_{i}, \quad r \mapsto\left(r_{1}, \ldots, r_{n}\right),
$$

is a ring monomorphism. Since $R_{i}=S_{i}^{-1} R$ is a division ring (Theorem 3.5 ), $R=S_{i} \coprod \mathfrak{a}_{i}$, by Lemma 3.4. Clearly, $\mathfrak{a}_{i}=\left\{r=\left(r_{1}, \ldots, r_{n}\right) \mid r_{i}=0\right\}$. Each element $s^{\prime}$ of the set $\mathcal{C}_{i}^{\prime}$ has the form $\left(0, \ldots, 0, s_{i}^{\prime}, 0, \ldots, 0\right)$ with $s_{i}^{\prime} \in R_{i}^{*}$. Then clearly, $s^{\prime} \mathfrak{a}_{i}=0$ and so $C_{i}^{\prime} \subseteq S_{i, c}$. To show that the equality $S_{i}^{\prime}=S_{i, c}$ holds it suffices to show that every element $s \in S_{i} \backslash C_{i}^{\prime}$ does not not belong to $S_{i, c}$. Fix $s$ such that $s \in S_{i} \backslash C_{i}^{\prime}$. Then there is an index, say $j$, such that $j \neq i$ and such that $s_{j} \neq 0$ in $s=\left(s_{1}, \ldots, s_{n}\right)$. Then $s \cdot \mathcal{C}_{j}^{\prime} \neq 0$ (Corollary 3.6) but $\mathcal{C}_{j}^{\prime} \subseteq \mathfrak{a}_{i}$. Therefore, the element $s$ does not belong to $S_{i, c}$.

\section{Acknowledgements}

The work is partly supported by the Royal Society and EPSRC. 


\section{References}

[1] V. V. Bavula, The algebra of integro-differential operators on an affine line and its modules, J. Pure Appl. Algebra, 217 (2013) 495-529. Arxiv:math.RA: 1011.2997.

[2] V. V. Bavula, The largest left quotient ring of a ring, Arxiv:math.RA:1101.5107.

[3] V. V. Bavula, New criteria for a ring to have a semisimple left quotient ring. Arxiv:math.RA:1303.0859.

[4] V. V. Bavula, Left localizations of left Artinian rings, Arxiv:math.RA:1405.0214.

Department of Pure Mathematics

University of Sheffield

Hicks Building

Sheffield S3 7RH

UK

email: v.bavula@sheffield.ac.uk 